\title{
NORMA IN LINGUISTICA E SOCIOLINGUISTICA E INCONGRUENZE TRA NORMA E USO NELL'ITALIANO D'OGGI
}

Questo lavoro consta di due parti. Nella prima (par. 1, 2,3,4) vorrei tentare una definizione di diversi tipi o aspetti della normatività linguistica, nella seconda (par. 5) vorrei analizzare come i diversi tipi di norma hanno agito e agiscono nell'ambito della lingua italiana d'oggi.

1. Norma linguistica e norma sociolinguistica.

Ogni regola1 implicita o esplicita che privilegia una forma piuttosto che un'altra è in sé norma, nel senso che indica qual'è o dovrebbe essere la scelta da compiere tra possibilità diverse.

Per poter distinguere tipi diversi di normatività conviene innanzi tutto prestare attenzione ai tipi diversi di variabilità che si presentano in un sistema linguistico. Un tipo di regole fondamentali sono quelle che indicano la variante da scegliere rispetto ad un insieme di altre possibili, ma che non appaiono di fatto nella pratica linguistica. A livello fonologico ${ }^{2}$ sono quelle regole che indicano innanzi tutto i fonemi di una lingua. Questi sono definiti sia dai tratti che li oppongono tra di loro, sia da tratti che li caratterizzano in sé, o meglio rispetto ad altre possibilità astratte e non reali. Cosí, per esempio, fa parte delle regole dell'italiano non solo la precisazione che la /b/ sia sonora, altrimenti si neutralizzerebbe con la $/ \mathrm{p} /$, ma anche la regola per cui essa deve essere bilabiale e non labiodentale come la /v/ o la regola per cui la $/ \mathrm{t} /$ e la /d/ italiane sono dentali e non alveolari come, per esempio, in inglese.

Un altro tipo di regole che precisano il reale rispetto al possibile sono quelle che indicano le varianti allofoniche che vengono determinate dal contesto fonetico quando le unità fonematiche vengono distribuite nella catena fonosintattica. E cosi abbiamo regole che impongono le realizzazioni di [n] come [n] in [venga] e come [n] in [dente].

1 Per "regola" non si intende qui nulla di prescrittivo, ma solo, per il momento, l'indicazione delle forme invalse all'interno della comunità linguistica, interiorizzata dal parlante a livello inconscio, senza la quale non vi sarebbe conoscenza condivisa del sistema linguistico e che permette la codificazione e la decodificazione linguistica.

2 Gli esempi scelti saranno d'ora in avanti principalmente tratti, per semplicità, a livello fonologico, ma la normatività copre ovviamente tutti i livelli del sistema. 
Le forme che queste regole indicano non hanno alternative, o varianti, invalse, cioè reali, ma solamente varianti teoriche, che esistono in potenza nel novero astratto delle forme linguistiche possibili. Per i fonemi sono forme possibili nell'ambito dei suoni che l'uomo può produrre ed usare. Per gli allofoni sono le forme possibili che il contesto può generare attraverso le possibili assimilazioni con i suoni con cui un fonema viene a contatto nella catena fonica. Altre possibilità astratte che potrebbero produrre varianti candidate alla concorrenza, sono tutte le variazioni individuali lungo la linea dell'analogia e dell' introduzione di forme nuove. Queste regole fissano perciò le forme contro la concorrenza di quelle che l'innovazione potrebbe introdurre.

La caratteristica fondamentale di queste regole è che esse sono categoriche e non ammettono un'applicazione statistica. Una forma, in un determinato contesto, o si usa o non si usa. Il loro scopo è quello di arginare l'intervento dell'individualità che porterebbe all'introduzione di varianti possibili.

Quando l'innovazione individuale o l'influenza di altri sistemi intacca questa difesa, possiamo avere la penetrazione di forme alternative, che possono espandersi nel sistema e riscuotere un consenso nella comunità linguistica tale per cui possono anche diffondersi in più idioletti, fino a valicare a volte barriere sociali e geografiche. In questo caso abbiamo varianti non più possibili o astratte ma reali, con indicazioni di preferenza. All'interno della comunità linguistica possono operarsi spaccature che portano ad un consenso solo parziale su una forma (variazione geografica o di gruppo etnico, di sesso, di età ed altri possibili). Per lo stesso parlante due forme antagoniste possono coesistere in contesti extralinguistici diversi (variazione stilistica o di registro). L'accettazione o occorrenza di queste varianti reali in conflitto sono a loro volta rispecchiate da regole invalse, che di fatto razionalizzano la convivenza di forme antagoniste.

Le regole del primo tipo sono disegnate per operare contro la variazione possibile, quelle del secondo tipo, non condivise da tutti i membri della comunità, sono disegnate per regolare la variazione in atto, dopo che le prime sono state in qualche modo violate dall'intervento dell'innovazione.

Vorremmo chiamare le prime regole linguistiche e le seconde regole sociolinguistiche. Le prime descrivono fatti di lingua senza tener conto né di parametri sociali (come la classe socio-educativa, il gruppo geografico, il sesso, l'età, la professione, il gruppo etnico o altri), né di condizionamenti legati alle caratteristiche sociali di un'interazione linguistica.

Le prime sono categoriche e indicano l'occorrenza necessaria di forme a volte condizionata da fatti interni al sistema: dato un determinato contesto paratattico, come per esempio il contesto fonetico per gli allofoni, abbiamo necessariamente l'occorrenza di una certa forma. Le seconde sono invece variabili, indicano cioè una tendenza statistica all'occorrenza della forma, e tale occorrenza non può essere spiegata in termini interni al sistema linguistico. 
Le regole sociolinguistiche, o regole variabili, sono state solo recentemente introdotte nella descrizione linguistica. Il problema che cercano di risolvere è sempre stato ben noto ai linguisti. Se, per esempio, esaminiamo a fondo la prima definizione di norma data da Coseriu, si vede come nella sua trattazione vi sia già esplicita l'ammissione del valore delle regole sociolinguistiche, come noi le abbiamo definite. Mentre per tutta la scuola strutturalista e per la linguistica trasformazionale viene negata completamente la possibilità di una regolarità della variazione, in Coseriu si fa strada, sia pur timidamente, il concetto di norma parziale:

“... in realtà, esistono varie norme parziali (sociali, regionali) poiché la norma, per la sua stessa natura, è sempre meno generale del sistema“ (Coseriu 1952, p. 66 della trad. it.).

Coseriu torna spesso, nella sua discussione teorica, sulla "norma parziale", anche se non la include nella sua teoria linguistica descrittiva, come nessun linguista della scuola strutturalista o d'altra scuola prima di Labov ${ }^{3}$.

Incidentalmente è interessante la distinzione che Coseriu fa tra "normale" $\mathrm{e}$ "corretto". Ciò che è normale rientra nel sistema e nella norma. Ciò che è corretto è oggetto di regole che non sono di norma, ma di prescrizione. Si tratta proprio della distinzione tra norma linguistica e norma sociolinguistica, tra invarianti ${ }^{4}$ e varianti: dove il termine "normale" sta per giusto vs. errato, cioè il contrario di forma aberrante in quanto inesistente, mentre "corretto" indica una forma migliore di un'altra o di altre che esistono, ma che sono in qualche modo peggiori:

Nogliamo chiarire inoltre che non si tratta della norma in senso corrente, stabilita ed imposta secondo criteri di concretezza e di valutazione soggettiva di quel che viene espresso, bensì della norma obiettivamente constatabile in una lingua, la norma che seguiamo necessariamente se vogliamo essere membri di una comunità linguistica, e non di quella secondo la quale si riconosce nella stessa comunità, se "parliamo bene" $\mathrm{o}$ in modo esemplare. Constatando la norma cui ci riferiamo, si constata come si dice e non si indica come si deve dire: i concetti che, riguardo ad essa, si oppongono l'un l'altro sono "normale" e "anormale" e non "corretto" e "scorretto". Il fatto che le altre norme possono coincidere non ci interessa qui: bisogna segnalare tuttavia che molte volte non coincidono, dato che la "norma normale" precede la "norma corretta", essendo sempre anteriore alla propria codificazione“. (Coseriu 1952 p. 76 della trad. it.).

\section{Per una tassonomia della variazione.}

Vorrei proporre qui una tassonomia della variazione linguistica. L'occorrenza di varianti può essere correlata o non correlata statisticamente con fatti interni o esterni al sistema.

3 Si veda, per esempio, Coseriu 1952, trad. it. 1971. Per un esame critico recente e approfondito della teoria di Coseriu su norma e sistema, si veda Lara 1983.

4 Per "invariabili" si intende qui quelle forme che non possono variare. Dato un contesto $x$, il sistema stesso fa prevedere quale variante apparirà, essa cioè è condizionata all'interno del sistema, dalla cooccorrenza con altre forme, ma deve occorrere necessariamente, una volta specificate le cooccorrenze. 


\section{VARIANTI CORRELATE}

1. CON FATTI INTERNI AL SISTEMA: allofoni.

2. CON FATTI ESTERNI AL SISTEMA:
[Varianti geografiche
[Varianti di classe
a) Varianti [Varianti di età
interpersonali [Varianti di gruppo etnico
[Varianti di sesso

b) Varianti intrapersonali: varianti stilistiche correlate con la formalità.

II. VARIANTI NON CORRELATE: Variazione libera.

La parte I.1 di questo schema rientra in quello che Coseriu ha definito sistema e norma. La parte II è minima ed è quella a cui dall'introduzione delle regole variabili in poi è stato relegato il concetto di variante libera che prima ricopriva invece tutta l'area indicata qui con I.2 (a e b). Quest'area è quella che è oggetto oggigiorno degli studi di macrosociolinguistica. Da quando è stata dimostrata la sistematicità della correlazione esterna al sistema, che si può descrivere prendendo in considerazione $i$ parametri extralinguistici, la variazione libera si è ridotta a quei casi molto rari in cui due (o più) varianti non hanno alcuna associazione di area o gruppo o situazione che ne determini la preferibilità in alcuni casi. In questo caso le varianti sono interscambiabili e libere da condizionamenti. Un esempio può essere la tendenza sporadica alla perdita dello stato fonematico delle vocali medie $e$ e $o$ in aree diverse da quelle centrali, per cui le due varianti aperta e chiusa:

hanno nel diasistema... negli usi sociolinguistici dell'intera nazione, uno scarso rendimento funzionale, quasi ridotto - in pratica - a variazioni libere, o diafoniche, determinate da sostrati ed astrati particolari, da analogie e dissomiglianze e da ipercorrettismi "stilistici" individuali. (Canepari 1979 , p. 195).

3. Norma sociale e norma prescrittiva.

La norma sociolinguistica copre tutta l'area del nostro schema. E'quella norma che, come abbiamo detto, regola la scelta tra varianti esistenti in competizione tra di loro.

Se consideriamo le varianti in competizione in diacronia, esse possono essere endogene e esogene. Le varianti esogene provengono da altri sistemi linguistici e si sono introdotte per contatto. Le altre sono innovazioni nate all'interno del sistema linguistico in questione che hanno avuto una fortuna solo parziale. E' inevitabile che sia le une che le altre vengano prima o poi dotate di una valenza di prestigio, negativa o positiva. L'italiano regionale è carico, come tutte le lingue, di variänti eso- 
gene provenienti dal sostrato dialettale: un esempio per tutti è il raddoppiamento di $/ \mathrm{b} / \mathrm{e} \mathrm{di} / \mathrm{d}_{3} /$ in posizione intervocalica, proveniente dal sostrato meridionale. L'italiano standard di origine toscana, il cosiddetto "italiano emendato", incontrandosi nelle aree meridionali col sostrato, ha ricevuto, tra le molte altre, questa variante esogena. In Toscana, però, questo stesso italiano emendato era stato costruito, a sua volta, escludendo varianti endogene come, per esempio, la $[/]$ per $[\mathrm{t} /]$ in posizione intervocalica, la [ts] per [s] dopo nasale é liquida, la [3] per [d३] e la gorgia.

Ogni variante avrà una sua fortuna fra $\mathrm{i}$ parlanti in misura diversa, sarà sentita da alcuni come accettabile ed usata di fatto, da altri no. Gli stessi parlanti potranno accettare anche una variante in competizione con un'altra accettata da tutti, in contesti socioliguisticamente diversi, oppure accettare di fatto, ma non sempre, che parlanti di area geografica o di gruppo diverso, usino varianti diverse dalle proprie. ${ }^{5}$

Tuttavia, la tendenza alla pluralità delle forme non opera indisturbata. La tendenza contraria è altrettanto forte: quella verso la scelta che privilegia una forma a scapito delle altre. La spinta fondamentale verso tale scelta è un'esigenza cognitiva profonda, quella che garantisce la formazione del pensiero astratto e del linguaggio che in parte lo rappresenta. E' un bisogno cognitivo di semplificare ciò che è in natura plurimo e complesso. E' lo stesso che ci porta alla scelta dei tratti distintivi che ci permettono di chiamare "gatto" una rappresentazione mentale che è definita solo dal comune denominatore scelto a definire tutti gli oggetti della classe. Lo stesso vale per la formazione dell'entità mentale e non fisica che chiamiamo fonema. Seguendo lo stesso istinto fondamentale, ogni parlante tende, se può, a scegliere nel caso delle varianti condizionate da fattori esterni al sistema, una variante come rappresentativa $o$ archeotipica di tutte le altre.

Questa scelta non può che essere arbitraria, perché in sé le varianti non sono più o meno buone funzionalmente in termini assoluti. In questo processo scattano proiezioni di valore che investono più o meno pesantemente le varianti. Si possono proiettare valenze estetiche, per cui una forma è sentita come più "bella" di un'altra; valenze di correttezza, per cui una variante è "giusta" o "sbagliata"; valenze funzionali $o$, infine, francamente sociali ${ }^{6}$. Si tratta di razionalizzazioni di una scelta in sé arbitraria, che va dal complesso verso il semplice, dalla pluralità verso l'unicità, verso la forma archeotipica. Non è un caso naturalmente che nella scelta della forma privilegiata abbia un ruolo molto importante la proiezione dei pregiudizi sociali sulle forme linguistiche. Dato che gli stereotipi sociali sono di primaria importanza nella mente degli individui, essi sono una buona fonte di proiezione di valore che discriminano tra le forme linguistiche legate ai diversi gruppi sociali che le usano. Queste proiezioni possono appunto essere più o meno razionalizzate poi in termini di "bontà", "bellezza" o "purezza".

5 Per la classificazione della variazione vista da diversi punti di vista, si veda Bell (1976, p. 33 e ss.).

6 Si veda per queste proiezioni Galli de’ Paratesi 1975 (Cap. III) e 1975. 
In pratica la spinta cognitiva che va verso la scelta di una forma come archeotipica e rappresentativa rispetto alle altre funzionalmente equivalenti, tende a compiere una operazione semplificatrice, tende a ridurre le varianti ad una sola, producendo regole categoriche laddove vi dovrebbero essere regole variabili e appiattendo i parametri di variazione. Un esempio di questa operazione è il fatto che nella formazione della norma si tende a preferire lo scritto al parlato, tendendo ad eliminare un parametro di variazione essenziale (si veda più avanti par. 4 e 5 ).

La norma, dunque, si insinua sempre spontaneamente dove ci sono più varianti non specializzate e per lo più si insinua come giudizio di valore proiettivo, che può essere più o meno condiviso dai membri di una comunità linguistica. Quando un giudizio è abbastanza condiviso, raccoglie cioè una sufficiente fortuna sociale, esso diventa regola, che può venire interiorizzata più o meno consciamente dai parlanti.

Le regole nascono quindi prima di tutto come delle norme di fatto, tacite e largamente se non interamente condivise, accettate cioè dalla maggioranza dei membri di una società. Questo costituisce la norma sociale, "sedimentata" come diceva Asco$\mathrm{li}^{7}$, la norma a posteriori ${ }^{8}$, che può essere descritta dai linguisti disposti ad analizzare l'uso di fatto.

Avviene poi che, generalmente, nelle società la cui lingua è trascritta, vi sia un'attività volta alla stesura di una norma prescrittiva. Tale norma è stilata o diffusa a volte a cura di istituzioni, come accademie, (come, per esempio, in Francia) o emittenti radiofoniche, come la $\mathrm{BBC}$ in Inghilterra, che tra le due guerre affidò la formazione dei propri annunciatori ad un alunno di Jones, Lloyd, o come la RAI, che nel passato aveva corsi di formazione per gli annunciatori ${ }^{9}$.

Altre volte la norma prescrittiva viene stilata con l'intervento di studiosi: è questo il contributo reso da Jones alla codificazione della Received Pronunciation, quello di Webster per l'inglese americano ${ }^{10}$, di Ben Yehuda per l'ebraico moderno in Israele e dei linguisti del Circólo di Praga per il ceco ${ }^{11}$.

La norma prescrittiva generalmente vuole essere nelle intenzioni un'esplicitazione della norma sociale, di solito quella della classe istruita. Intende cioè essere una norma a posteriori rispetto alla sedimentazione di fatto che vuole solo descrivere. In realtà, anche quando è veramente posteriore ad una sedimentazione, essa tende ad essere autoavverantesi, cioè il fatto stesso che venga stilata ne aumenta il consenso. Ma, ancora più importante, essa non può non essere una riduzione della pluralità e della complessità della norma di fatto, poichè appunto privilegia uno dei socioletti, quello a livello più alto di istruzione, lo scritto rispetto al parlato,

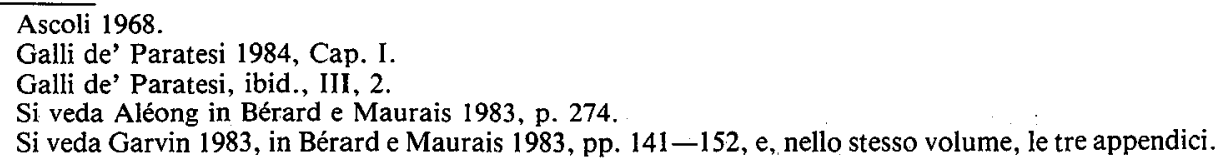


il registro più formale rispetto a quelli informali, $i$ gruppi etnici o regionali non colpiti da discriminazione, i gruppi di età meno giovani rispetto ai più giovani. Ancora una volta abbiamo un andamento dal complesso al semplice, dal pluralismo all'unico e perciò l'intervento dell'arbitrarietà.

Dunque se la sedimentazione della norma sociale è una riduzione rispetto all'uso linguistico di fatto, il passaggio dalla norma sociale a quella prescrittiva è un'ulteriore riduzione, che, attraverso proiezioni di giudizi di valore negativi, riduce o tenta di ridurre il polimorfismo. Aléong (1893) ha messo in luce molto bene il valore riduttivo della norma prescrittiva:

\begin{abstract}
la nostra definizione mette in evidenza il carattere relativo di qualsiasi giudizio di valore e lascia intravedere la possibilità dell'esistenza di più di una norma linguistica... Tuttavia, anche riconoscendo l'esistenza di diverse norme linguistiche, bisogna constatare che, nel suo senso abituale, questo termine designa una varietà di lingua che, in un certo momento, si impone ed è imposto da tutto un apparato descrittivo come la lingua di riferimento, sulla base della quale si devono misurare tutti i comportamenti linguistici. E' la lingua corretta che, per definizione, relega tutte le altre forme possibili nell'ambito dell'errore... poichè la norma rappresenta una selezione tra le forme reali o possibili, bisogna concludere che essa ha un valore arbitrario. (p. 261).
\end{abstract}

4. Norma a posteriori e norma a priori.

Finora, nel parlare di una norma prescrittiva, ci siamo tenuti al caso in cui vi fosse un largo consenso intorno ad una norma sociale invalsa e si tentasse di trascriverla e diffonderla ulteriormente. Questo è il caso del francese e dell'inglese sia britannico che americano. In tutti e tre questi casi si sono verificate le condizioni storiche necessarie perché la norma sociale sedimentasse, cioè la formazione di uno stato unitario che, attraverso soprattutto la scuola, diffondesse la varietà delle classi istruite come buona. L'accademia francese, Jones e Webster sono stati, con ruoli diversi, dei trascrittori di un uso linguistico accolto da un largo consenso.

Sono molto più frequenti, però, situazioni in cui la mancanza o la formazione appena recente di uno stato unitario non ha permesso la sedimentazione di una norma sociale a livello nazionale. Abbiamo allora interventi in cui la norma viene scelta prima della sua sedimentazione e viene imposta, a volte addirittura non solo prima ma contro il consenso, nel tentativo di far accadere artificialmente ciò che, per lingue come il francese e l'inglese, è accaduto nel corso di secoli.

Questo tipo di norma a priori è molto comune nei paesi ex-coloniali e rimane da dimostrare che sia candidata al successo. Un caso positivo da citare a questo proposito è senz'altro quello di Israle, dove l'elaborazione dell'ebraico classico compiuta da Ben Yehuda ha avuto, per le necessità di fatto delle circostanze storico-sociali del paese, cioè l'assenza di una lingua comune a tutti i cittadini, un succeso totale. Un 
altro esito positivo di un intervento di linguisti, che però era solo parzialmente a priori, è il caso citato sopra del $\mathrm{ceco}^{12}$.

L'Italia rappresenta un caso particolare in questo senso. Privata per secoli di un'unità politica nazionale, quando l'ha finalmente raggiunta, ha visto la nascita di uno sviluppo economico eccentrico rispetto sia alla collocazione geografica della capitale, sia al luogo di origine della lingua nazionale. Per secoli non ha visto il sedimentarsi di una norma sociale di fatto della lingua parlata. Da questo è derivato un profondo malessere, che ha spinto molti autori a indicare norme da scegliere a priori nell'illusione di innestare una norma sociale. Spesso le forme di lingua scelte per queste operazioni, sempre augurate piuttosto che compiute, ma sistematiche, non erano neppure realmente esistenti, come il mitico toscano del duecento o il volgare illustre di Dante, ma oggetto di nostalgie e di vagheggiamenti. Ancora al momento dell'unificazione abbiamo un'altra scelta, più a tavolino che nell'intervento attivo, che non aveva molto a che fare coi fatti o con una sedimentazione che mancava: il manzoniano fiorentino parlato che avrebbe dovuto essere scelto come norma di tutte le regioni.

La sedimentazione preconizzata dalla preveggenza di Ascoli in quel frangente, ha, in effetti, avuto luogo da allora e assistiamo ormai alla presa di coscienza della formazione, ancora agli inizi, di una forma di fatto parlata, anche se parziale.

Tuttavia, secoli di vuoto di norma sociale nel parlato avevano lasciato uno spazio in cui si era pesantemente inserita una norma a priori, il dominio incondizionato, a livello prescrittivo, dell'italiano di origine toscana che, nella letteratura normativa, aveva fatto da padrone specialmente nell'ultimo secolo. Norma prescrittiva da noi aveva finito coll'avere ben poco a che fare con l'uso di fatto, ed ora questo divario rende ormai i nostri manuali descrittivi molto largamente incongruenti non solo con l'uso orale, ma anche con un nuovo uso scritto che è di fatto emerso.

5. Incongruenze ed evoluzioni di norma e uso nell'italiano di oggi.

La prescrizione sta alla norma di fatto (o, come s'è visto, alle norme di fatto, perchè l'uso linguistico varia a seconda dei parametri sociali) come una riduzione $e$, in effetti, un impoverimento, che è il risultato di scelte arbitrarie che scartano come "non buone" forme che in realtà sono usate.

Nella ricerca di una forma archeotipica viene favorito pesantemente lo scritto rispetto al parlato. Ciò avviene innanzi tutto perché lo scritto è legato al prestigio dell'istruzione. Inoltre lo scritto ha il vantaggio di costituire un corpus fisicamente tangibile e accessibile, che può essere analizzato e descritto ed a cui ci si riferisce quando si hanno dei dubbi. Non è un caso che gli studiosi del Circolo di Praga aves-

12 Per operazioni politiche dirigistiche di questo tipo si vedano, per esempio: Paquette 1983, Fishman 1983, Rondeau 1983 in Bérard e Maurais 1983. 
sero indicato il corpus letterario degli ultimi cinquant'anni come il punto di riferimento della norma che intendevano stilare. Ma vi è un'altra ragione più strettamente cognitiva che porta alla preferenza verso lo scritto come norma ed è il fatto che ciò che si tende a scegliere come norma è preferibilmente una forma non marcata rispetto ad una forma marcata, esplicita e completa, rispetto ad una formulazione ellettica: lo scritto per sua natura è più esplicito, meno anacolutico del parlato. Il fare, però, dello scritto una regola prescrittiva a scapito del parlato porta a conseguenze gravi: un esempio in questo senso è la regola normativa che dà come non grammaticale una forma come "a me mi piace" invece di "mi piace" o "a me piace". La forma stigmatizzata come errore è in realtà una forma marcata, con una focalizzazione che pone enfasi sul pronome. Un processo molto naturale in ogni lingua, tendenzialmente più spesso presente nel parlato che nello scritto. In realtà la forma originalmente marcata, con la ripetizione del pronome, è usata ormai comunemente nel parlato di tutte le classi sociali ed è entrata comunemente nello scritto.

Il divario parlato-scritto possiede una caratteristica molto saliente: che aumenta, a volte a dismisura, in diacronia. Lo scritto, infatti, proprio perché tale e perché ha la funzione di corpus di riferimento, ha un'evoluzione molto più lenta. Il parlato invece ha una deriva inarrestabile. Quando una norma descrittiva ha fissato certe forme scritte come buone, anche se esse coincidono con quelle dell'orale, non è infrequente che risultano ben presto obsolete riguardo all'orale che, nel frattempo, è stato trascinato altrove dall'effetto di deriva. Questo è il caso, per l'italiano, di una larga parte del nostro vocabolario della lingua scritta e di regole, ormai incongrue, come loro, per il pronome dell'oggetto indiretto plurale, invece di gli.

Per secoli l'italiano, come s'è detto, non ha avuto una norma di riferimento parlata a livello nazionale. Esclusi pochi al di fuori della Toscana, gli italiani che sapevano leggere e scrivere non usavano l'italiano per parlare, ma un dialetto locale a volte molto lontano dalla lingua scritta. L'italiano scritto, non avendo una controparte orale con cui confrontarsi in termini di evoluzione diacronica, aveva potuto sottrarsi quasi completamente alla deriva di cui s'è parlato. E' stato infatti, e tende ancora ad essere, una lingua cristallizzata, molto di più di quanto non lo fosse, per esempio, l'inglese scritto, che ha avuto sempre un confronto continuo con l'inglese parlato. Quando la fonte del parlato manca, lo scritto rimane avulso dalla realtà e fortemente frenato nella sua evoluzione diacronica. Adesso che la quasi totalità degli italiani è italofona in qualche misura, la lingua italiana, pur nelle sue coloriture regionali, esiste e preme per far entrare nello scritto forme ormai invalse. C'è qualcosa di innaturale che è familiare a molti italiani, nel non scrivere "a me mi piace", "gli ho detto" nel senso di "ho detto loro", "c'ho fame" congiuntivo per chi usa l'indicativo in frasi come "credo che viene", eccetera.

13 Che "ch'o" per "ho" non sia solo una variante stilistica da evitare, è dimostrato da casi in cui il suo uso è obbligatorio e non alternativo, come nella risposta alla domanda "Hai un libro?" (Sabatini 1981). Tutte queste forme, che sono bloccate dalla norma, ma usate ampliamente nel parlato e anche nello scritto, sono molto antiche. Per la loro storia e diffusione si veda Sabatini 1986. 
Non solo ormai l'orale preme per fare entrare le sue forme nello scritto, ma ha anche trovato il modo in cui farlo. I giornali, per esempio, hanno ormai fatto nascere un italiano scritto non letterario o accademico in cui l'italiano orale si rispecchia largamente e di fronte al quale la norma prescrittiva delle nostre grammatiche di "buon italiano" è fortemente incongruente.

Un'altra incogruenza tra norma prescrittiva e norma di fatto è la "toscanità" della prima nei confronti della seconda. In questo senso l'incongruenza nasce dalla particolarità di essere la nostra norma prescrittiva, come s'è detto, a priori nella realtà della lingua in uso. La norma prescrittiva, com'è descritta nelle grammatiche normative e nei prontuari di pronuncia, auspicava e incoraggiava l'avvento del toscano, variamente descritto come "colto", "emendato" o altro. Cosí troviamo nelle grammatiche, per esempio, codesto o l'uso anche orale del passato remoto secondo l'uso toscano, che sono ben lungi da essere diffusi a livello nazionale, né sembrano candidati ad esserlo. $\mathrm{E}$, a livello di pronuncia, troviamo una spaccatura tra quelle caratteristiche del toscano che erano registrate nella grafia e che si sono in effetti diffuse e quelle non registrate dalla grafia, ma raccomandate come normativamente buone, che sono rimaste lettera morta fuori della Toscana, laddove non erano condivise dal sostrato locale. E'il caso, tra i molti altri, della distinzione fonematica tra /e/ e $/ \varepsilon /, / \mathrm{o} / \mathrm{e} / \mathrm{/} / \mathrm{/ \textrm {s }} / \mathrm{e} / \mathrm{z} /, / \mathrm{ts} / \mathrm{e} / \mathrm{dz} /$. Per quanto riguarda le due paia di opposizioni delle vocali medie, laddove la distinzione fonematica non esisteva (come, per esempio, in Calabria, Trieste e Sardegna) non è penetrata. Laddove essa esisteva, ma con distribuzione diversa, non abbiamo una evidente vittoria della distribuzione toscana. Per la $s$ e la $z$ abbiamo lo status quo e, casomai, appaiono vincenti due forme settentrionali, la [dz] iniziale, come per esempio in zio e la [z] intervocalica, come in meraviglioso ${ }^{14}$. Il raddoppiamento iniziale, obbligatorio nella forma prescrittiva, non appartiene all'uso di fatto nell'Italia settentrionale.

Con la nascita della norma orale di fatto o con l'inizio della sua sedimentazione a cui abbiamo assistito negli ultimi anni, s'è eliminata la fonte delle incongruenze maggiori che il divario tra norma prescrittiva e uso presentavano in italiano. Stiamo assistendo al tramonto della norma a priori. Ormai sarebbe difficile concepire una grammatica dignitosa dell'italiano, che volesse imporre una forma di lingua e non registrare quelle forme che raccolgono maggior consenso. Le descrizioni dell'italiano standard o normativo, come, per esempio, quelle di Canepari (1979 e 1980), contengono anche le varianti regionali, non tanto come indicazione di norma per tutti, ma come descrizione delle norme di fatto alternative. Col sorgere della norma sovraregionale di fatto, destinata a coesistere con quelle locali, dovrà nascere una norma a posteriori, non più toscaneggiante a tutti i costi, ma nazionale, con un'interazione tale tra parlato e scritto da rendere quest'ultimo accettabile da chi l'italiano, sia pur regionale, lo parla, non più isolata rispetto all'effetto di deriva diacronica, ma situata nel divenire normale di una lingua in uso.

14 Si veda Lepschy e Lepschy 1977 , p. 80. 
Aleong, S., "Normes linguistiques, normes sociales, une perspective anthropologique" in Bérard e Maurais; 1983.

Ascoli, G. I., Scritti sulla questione della lingua, a cura di C. Grassi, Torino; 1968.

Bell, R., Sociolinguistics. Goals, Approaches and Problems, Londra; 1976.

Bérard, E., Maurais, J., (a cura di) La norme linguistique, Quebec; 1983.

Canepari, L., Introduzione alla fonetica, Torino; 1979.

Canepari, L., Italiano standard e pronunce regionali, Padova; 1980.

Coseriu, E., Sistema, norma y habla, Montevideo; 1952, trad. it. "Sistema, norma e 'parole'" in Teoria del linguaggio e linguistica generale, Bari; 1971: pp. 19 -103 .

Galli de' Paratesi, Nora, "Analisi semantica delle opinioni linguistiche, un caso di sinestesia in senso lato" in Studi in onore di Giuliano Bonfante, Brescia; 1975: pp. 281-294.

Galli de' Paratesi, Nora, Lingua toscana in bocca ambrosiana, Bologna; 1985.

Garvin, P. L., "Le rôle des linguistes de l'Ecole de Prague dans le développement de la norme linguistique tchèque" in Bérard e Maurais; 1983.

Lara, F., "Le concept de norme dans la théorie d'Eugenio Coseriu“ in Bérard e Maurais; 1983.

Lepschy, A. L., Lepschy, G., The Italian language today, Londra; 1977, trad. it. La lingua italiana. Storia delle varietà d'uso e grammatica, Milano; 1981.

Paquette, J. M., "Procès de normalisation et niveau-registres de langue" in Bérard e Maurais; 1983.

Rondeau, G., "La normalisation linguistique, terminologie et technique au Quebec" in Bérard e Maurais; 1983.

Sabatini, F., "Linee di tendenza dell'italiano contemporaneo" in Lindgren L. (a cura di) La lingua italiana in Finlandia. Atti del I Congresso degli Insegnanti in Finlandia, Turku; 1980: pp. 73-91.

Sabatini, F., "L'italiano dell'uso medio: una realtà tra le varietà linguistiche italiane" in Gesprochenes Italienisch in Geschichte und Gegenwart a cura di G. Holtus e E. Radtke, Tübingen; 1986.

Povzetek

NORMA V LINGVISTIKI IN SOCIOLINGVISTIKI TER NESKLADJE MED NORMO IN RABO V ITALIJANŠČINI

Avtorica je za sodobno italijanščino prepričana, da je treba ob nekdanji in v opisnih ter šolskih slovnicah nasploh prevladujoči toskanski normi bolj upoštevati dejansko stanje v govorjenem jeziku. Znano je, da je italijanščina izrazito knjižni jezik, vendar jezikoslovje zadnjih desetletij le dopušča alternativne možnosti. Danes si ni več mogoče zamišljati slovnice, ki bi upoštevala samo toskansko rabo. Sociolingvistična norma je nadpokrajinska, pri čemer se ublaži ostra razlika med pisanim in govorjenim jezikom. 